\title{
Coexistence of pseudomorphic and coincidence phases in nickel monolayers
}

\author{
C. Schmidthals *, A. Enders, D. Sander, J. Kirschner \\ Max-Planck-Institut für Mikrostrukturphysik, Weinberg 2, D-06120 Halle, Germany
}

Received 21 July 1997; accepted for publication 2 October 1997

\begin{abstract}
We report a study of the structural properties of Ni monolayers on W(110) using low-energy electron diffraction (LEED), spot profile analyzing LEED (SPA-LEED) and scanning tunneling microscopy (STM) at room temperature. Below a coverage of 0.4 monolayers $(\mathrm{ML})$ we find a pseudomorphic $(1 \times 1)$ LEED pattern. Starting from $0.4 \mathrm{ML}$, LEED experiments show a $(8 \times 1)$ superstructure, followed by a $(7 \times 1)$ superstructure at a coverage of about $0.7 \mathrm{ML}$. These superstructures are interpreted as coincidence structures. A stripe pattern characteristic of the $(7 \times 1)$ structure is found with STM. STM indicates a partial transformation into the $(7 \times 1)$ coincidence structure: $\sim 10 \%$ of the first monolayer in the vicinity of surface defects remains pseudomorphic. Annealing the films leads to a lateral separation of the pseudomorphic and $(7 \times 1)$ coincidence structures, but not to the growth of one phase in preference to the other. (c) 1998 Elsevier Science B.V. All rights reserved.
\end{abstract}

Keywords: Coincidence structure; Low energy electron diffraction (LEED); Nickel; Scanning tunneling microscopy (STM); Tungsten

\section{Introduction}

In many heteroepitaxial systems the difference in the lattice spacings of the film and the substrate causes structural modifications of the growing film $[1,2]$. Theoretical work [3] on the growth of facecentered cubic (fcc) film materials on body-centered cubic (bcc) substrates show that either pseudomorphism or a distortion network should be observed once the bulk lattice constants of the materials involved and their interactions at the interface are given. Previous LEED studies [4] on the growth of $\mathrm{Ni}$ on a $\mathrm{W}(110)$ substrate have indicated that a transition from pseudomorphic growth to a $(8 \times 1)$ coincidence structure takes place at a coverage of about 0.4 monolayers (ML).

* Corresponding author. Fax: (+49) 345 5511223; e-mail: cord@mpi-halle.mpg.de
With increasing coverage, yet another transition to a $(7 \times 1)$ coincidence structure has been observed. In extension to these results we report on a combined study with LEED, STM and spot profile analyzing LEED (SPA-LEED). Our results show that the first complete Ni layer does not transform into a $(7 \times 1)$ structure, but that some patches of the film remain pseudomorphic with increasing coverage. We propose that the coexistence of a coincidence structure and the pseudomorphic phase can be explained by the action of defects such as substrate steps and holes in the film stabilizing the pseudomorphic phase.

\section{Experimental}

The films were deposited on an electropolished $\mathrm{W}(110)$ single crystal cut in the [110] direction to 
within $0.5^{\circ}$, giving a mean terrace width of about $30 \mathrm{~nm}$ as measured with STM and SPA-LEED. Initial cleaning was performed in a separate highvacuum chamber by heating to $1800 \mathrm{~K}$ for about $120 \mathrm{~h}$ in an oxygen atmosphere of $1 \times 10^{-6}$ mbar. After transferring the sample into an ultrahigh vacuum chamber with a base pressure of $6 \times 10^{-11} \mathrm{mbar}$, the crystal was finally cleaned by cycles of annealing at $2300 \mathrm{~K}$ in vacuum and heating to $1600 \mathrm{~K}$ in an oxygen pressure of $1 \times 10^{-6}$ mbar. This procedure led to a contamination level of about $0.5 \%$ of a monolayer (ML) of oxygen and carbon, as determined by Auger electron spectroscopy. $\mathrm{Ni}$ films were produced by thermal sublimation of an ultraclean $(99.999 \%)$ nickel rod heated by electron bombardment with growth rates of about $0.5 \mathrm{ML} \mathrm{min}^{-1}$. The pressure during deposition never rose above $5 \times 10^{-10}$ mbar. Coverages were determined by STM, leading to an absolute accuracy of better than $0.1 \mathrm{ML}$.

\section{Results and discussion}

Since our results on the Ni film structure up to a coverage of $0.7 \mathrm{ML}$ are in full agreement with previous studies [4], only a short description of the structural evolution in the submonolayer range will be given here. Throughout this paper, one monolayer corresponds to the packing density of the fcc Ni(111) plane. Below a coverage of about 0.4 ML, the LEED images are identical to the pattern obtained from the clean W(110) surface, indicating pseudomorphic growth in this coverage range. In the Nishiyama-Wassermann orientation [4], observed above $1 \mathrm{ML}$, the $\mathrm{Ni}(111)$ plane lies parallel to the $\mathrm{W}(110)$ plane and the $\mathrm{Ni}\langle 0 \overline{1} 1\rangle$ directions are parallel to the $\mathrm{W}\langle 001\rangle$ directions. This epitaxial orientation leads to a large strongly anisotropic strain in the pseudomorphic phase of $27 \%$ along the $\mathrm{W}[001]$ direction and a moderate strain of $3.7 \%$ in the $\mathrm{W}[\overline{1} 10]$ direction, as compared to the respective $\mathrm{Ni}-\mathrm{Ni}$ distances in bulk $\mathrm{Ni}(111)$. In contrast to $\mathrm{Ni}$ films grown on $\operatorname{Re}(0001)$ [7] and Fe films on W(110) [8], where the matching surface symmetries of the film and the substrate lead to an isotropic mismatch, the two-fold symmetry of the W(110) surface results in a highly anisotropic mismatch to the six-fold symmetric $\mathrm{Ni}(111)$ plane. Thus a tremendous strain energy builds up initially. Above $0.4 \mathrm{ML}$ the strain energy is lowered by forming a $(8 \times 1)$ coincidence structure where nine $\mathrm{Ni}-\mathrm{Ni}$ atomic distances correspond to eight $\mathrm{W}-\mathrm{W}$ atomic distances. In this configuration the tensile strain in the $\mathrm{Ni}$ film is reduced from $27 \%$ to $13 \%$ in the $\mathrm{W}$ [001] direction. Further Ni deposition leads to the formation of a $(7 \times 1)$ coincidence structure starting at a coverage of $0.7 \mathrm{ML}$. This is demonstrated in Fig. 1a, which shows the diffraction pattern of a $0.9 \mathrm{ML}$ thick film. The additional spots in the $\mathrm{W}[001]$ direction are separated by one seventh of the substrate spot distance. This diffraction pattern can be explained by the simple hardsphere model shown in Fig. 1b. Here, every tenth $\mathrm{Ni}$ atom coincides with every eighth $\mathrm{W}$ atom, thus leading to a correspondence of nine $\mathrm{Ni}-\mathrm{Ni}$ atomic distances to seven $\mathrm{W}-\mathrm{W}$ atomic distances, reducing the value of the strain further to a small compression of $-1.3 \%$. Direct measurements of the mechanical stress in the film [5] are in good agreement with this structural evolution. In the simple hard-sphere model, the 9/7 coincidence leads to a "wavyness" of the surface with a periodicy of $1.1 \mathrm{~nm}$ and an amplitude of $0.06 \mathrm{~nm}$, as indicated in Fig. 1b. This simple model seems to describe the film structure adequately, as evidenced by the STM image of Fig. 1c: at a coverage of $0.9 \mathrm{ML}$ the first layer is not yet completely filled and some holes in the first monolayer (grey) down to the substrate are represented as black patches. A periodic arrangement of stripes along W[001] can be seen on the surface of the first layer. The stripes are separated by $1.06 \mathrm{~nm}$, in very good agreement with the prediction of the $9 / 7$ coincidence model. Note that a coincidence of only every eighth $\mathrm{Ni}$ atom with every seventh $\mathrm{W}$ atom $(8 / 7$ coincidence) would lead to the same $(7 \times 1)$ diffraction pattern as in Fig. 1a. However, for a $8 / 7$ coincidence a periodicy of $2.2 \mathrm{~nm}$ results, in clear contradiction to our STM results. Thus, in contrast to recent results of corresponding systems like $\mathrm{Ni} / \mathrm{Re}(0001)$ [7] and $\mathrm{Fe} / \mathrm{W}(110)$ [8], we observe a contrast with STM even at submonolayer coverages. The height corrugation in Fig. 1c 

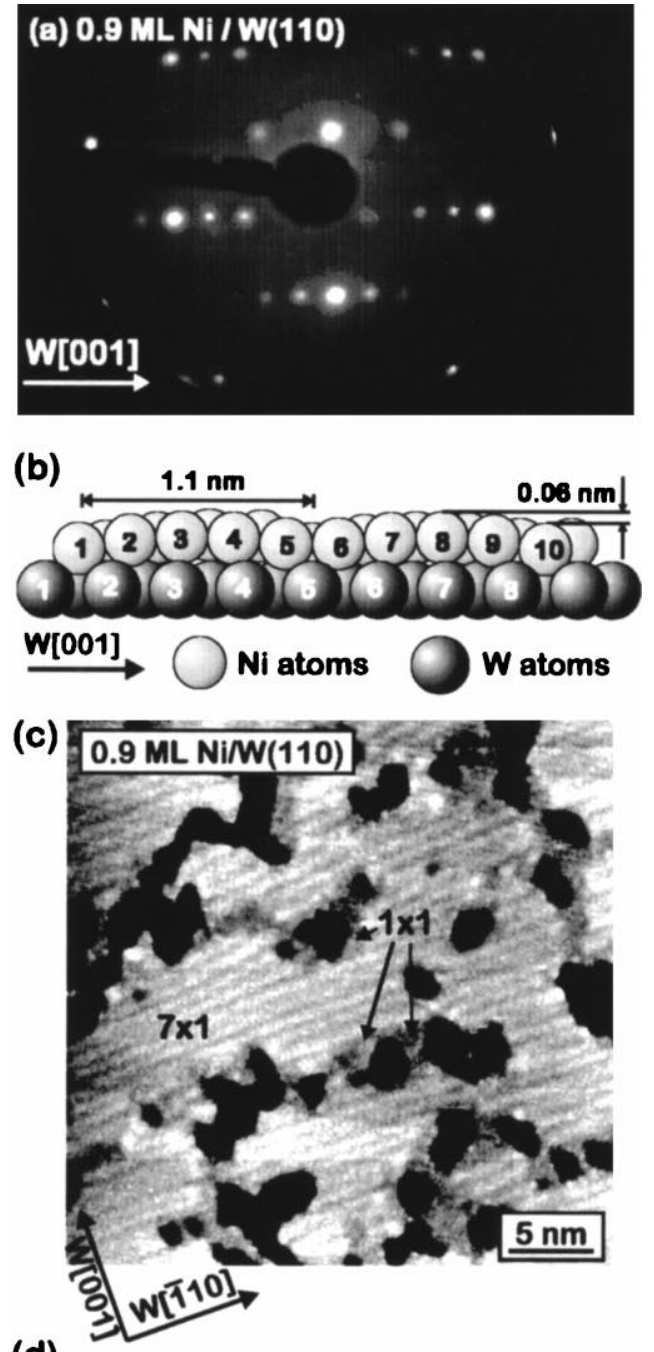

(d)

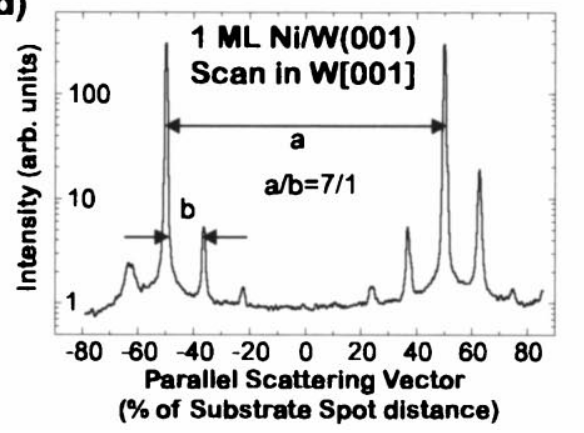

Fig. 1. (a) LEED image of $0.9 \mathrm{ML}$ Ni taken at $129 \mathrm{eV}$. The $(7 \times 1)$ superstructure spots are clearly visible. (b) Hard-sphere model for the first atomic layer of $\mathrm{Ni}$ on $\mathrm{W}(110)$. The coincidence structure leads to a "wavyness" of the $\mathrm{Ni}$ film with a periodicy of $1.1 \mathrm{~nm}$ and an amplitude of $0.06 \mathrm{~nm}$. (c) STM amounts to $0.006 \mathrm{~nm}$, which is a factor of 10 smaller than the predicted value of the hard-sphere model. Bearing in mind that the STM probes the local electronic density of states [6] and not the height of a surface, the smaller corrugation of the STM images as compared to the structural model should not be overestimated.

Now we turn to the origin of the dark grey patches around the black holes in the film in the STM image of Fig. 1c, marked by " $1 \times 1$ ". Auger electron spectroscopy indicates a contamination level below $1 \%$ of a monolayer. However, the dark grey areas amount to about $10 \%$ of the surface area. Therefore we rule out contaminations as an explanation for the observed STM contrast. Moreover, the exclusive occurrence of the darker patches of the annealed film on narrow terraces indicates a structural induced contrast mechanism, as discussed below. In order to check whether the dark grey areas have a different structure we used our SPA-LEED system, which probes the surface structure with a strongly increased sensitivity as compared to conventional LEED. A line profile along W[001] of a $1 \mathrm{ML}$ film is shown in Fig. 1d. Here the intensity of the diffracted beams is plotted as a function of the diffraction angle. The diffraction angle has been scaled so that the substrate spot distance becomes $100 \%$. The line scan proves that apart from the seven-fold superstructure and some diffuse background, no further structure can be detected. However, pseudomorphic areas of the film cannot be distinguished from the spots arising from the substrate without a quantitative intensity analysis, which we did not perform. Therefore we conclude that the darker grey patches seen in the STM images can be identified as pseudomorphic regions on the surface. Unfortunately, due to instrumental limitations, we could not obtain atomic resolution. Therefore, at this stage we cannot definitely rule out the possibility that the

image of $0.9 \mathrm{ML} \mathrm{Ni}$ on $\mathrm{W}(110)$. The stripe pattern indicates the "wavyness" of the $(7 \times 1)$ coincidence structure. Near the holes in the film (black) darker grey patches indicate pseudomorphic areas. (d) LEED intensity line scan in the W[001] direction. Apart from the $(7 \times 1)$ superstructure spots, no additional spots can be detected. Note the logarithmic scale of the intensity axis. 
dark patches consist of an unordered phase, the film atoms having no periodic arrangement. However, total energy considerations [8] suggest that the pseudomorphic configuration is favored over this possibility. The contrast between the $(7 \times 1)$ and the $(1 \times 1)$ phases seen with STM depends on the applied bias voltage and becomes strongest for positive sample bias voltages. This indicates a contrast mechnism originating from electronic effects. The large differences in the bond conditions in the $(7 \times 1)$ and the pseudomorphic patches could cause a different local density of states in each region, thus leading to the observed contrast. We conclude that for coverages between 0.7 and $1 \mathrm{ML}$ there is a coexistence of $(7 \times 1)$ coincidence structures and pseudomorphic areas on the surface.

In order to check whether the pseudomorphic phase is a result of hindered growth kinetics at $300 \mathrm{~K}$, we annealed the film shown in Fig. 1c for several minutes to $900 \mathrm{~K}$. In Fig. $2 \mathrm{a}$ and $\mathrm{b}$ two STM images of the same surface regions of a 0.9 ML Ni film are shown. Fig. $2 b$ shows a smallscale image of the area marked by a square in Fig. 2a. Dark patches can be seen on narrow terraces of the film, which are mainly oriented along W[001]. The high-magnification image in Fig. $2 \mathrm{~b}$ shows that the apparent lighter areas consist of an ordered $(7 \times 1)$ coincidence structure, as indicated by the stripe pattern, and that the dark patches represent the pseudomorphic $(1 \times 1)$ structure. Interestingly, annealing did not lead to the growth of one structural phase in preference account of the other, indicating that hindered growth kinetics are not responsible for the coexistence of both structural phases. While in the unannealed film both phases coexist on one terrace as patches with sizes of only a few nm (see Fig. 1c), after annealing each phase has agglomerated into areas of some $10 \mathrm{~nm}$ in size. Thereby, the coexistence of the pseudomorphic and the $(7 \times 1)$ coincidence structure is retained upon annealing. Now the pseudomorphic areas are limited to the upper and lower step edges of the substrate and to narrow terraces below about $70 \mathrm{~nm}$ in width. The patches are preferentially oriented along W[001], while the terrace width in the $\mathrm{W}[\overline{1} 10]$ direction does not seem to influence the formation of the
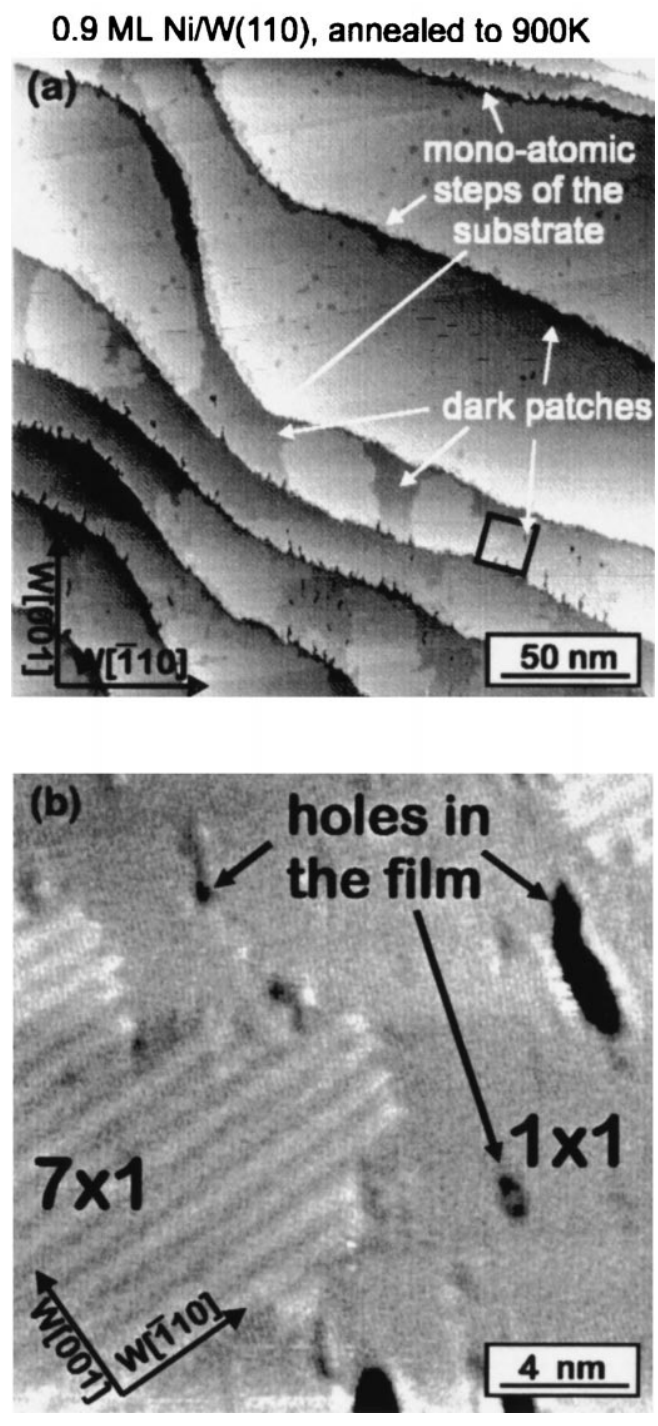

Fig. 2. STM images of a 0.9 ML Ni film on W(110). (a) Dark grey patches are preferentially located at substrate steps and on narrow terraces. The patches show a preferential extension along the $\mathrm{W}[001]$ direction. The surface region magnified in (b) is marked by a square. (b) Coexistence of the $(7 \times 1)$ coincidence structure indicated by the stripe pattern and the pseudomorphic area denoted " $1 \times 1$ ", appearing as a dark patch in (a). Note the rotated scan direction compared to (a) in order to improve the small contrast obtained from the coincidence structure.

pseudomorphic phase. A plausible explanation for the preferential location of the pseudomorphic patches on narrow terraces is proposed to be due 
to the strain energies involved. In the pseudomorphic phase, the anisotropic strain in the film amounts to almost 27\% along $\mathrm{W}[001]$ and only $3.6 \%$ along $\mathrm{W}[\overline{1} 10]$. Assuming that the substrate steps can stabilize the energetically unfavourable pseudomorphic phase, it becomes clear that above a certain terrace width the stabilizing interaction of the steps, given for example by inward relaxation $[9,10]$, is not sufficient to account for the tremendous strain energies. Along W[1110], however, no dependence on the terrace width is observed because of the smaller strain (smaller by almost a factor of 10) in that direction. On narrow terraces along $\mathrm{W}[001]$, the strongly strained pseudomorphic film can be stabilized, because at the terrace edge at least one atom can relax inwards, partially decreasing the strain energy. A similar relaxation mechanism has also been proposed to explain the oscillatory behavior of the lateral lattice parameter with increasing coverage in diffraction experiments [9]. The same mechanism was used as an explanation for the hindered coalescence of $\mathrm{Fe}$ islands grown on W(110) [10].

In conclusion, our STM images reveal the coexistence of pseudomorphic $(1 \times 1)$ and $(7 \times 1)$ coincidence structures in the first monolayer of $\mathrm{Ni}$ on W(110). In an extension of previous work [4], STM shows that annealing the films leads to a lateral separation of the pseudomorphic and coincidence regions, but not to the growth of one of the regions. The pseudomorphic areas are stabilized at surface defects such as substrate steps, and show a distinct preferential orientation along $\mathrm{W}$ [001] which can be explained by the strongly anisotropic strains in this phase.

\section{References}

[1] J.H. van der Merwe, D.L. Tönsing, P.M. Stoop, Surf. Sci. 312 (1994) 387.

[2] J.H. van der Merwe, J. Woltersdorf, W.A. Jesser, Mater. Sci. Eng. 81 (1986) 1.

[3] L.C.A. Stoop, J.H. van der Merwe, Thin Solid Films 94 (1982) 341.

[4] J. Kolaczkiewicz, E. Bauer, Surf. Sci. 144 (1984) 495.

[5] D. Sander, A. Enders and C. Schmidthals, J. Kirschner, Surf. Sci., this issue.

[6] C. Hamann, M. Hietschold, Raster-Tunnel-Mikroskopie, Akademie Verlag, Berlin, 1991.

[7] M. Stindtmann, M. Farle, T.S. Rahman, L. Benabid, K. Baberschke, Surf. Sci. 381 (1997) 12.

[8] E. Bauer, J.H. van der Merwe, Phys. Rev. B 33 (1986) 3657.

[9] J. Fassbender, U. May, B. Schirmer, R.M. Jungblut, B. Hillebrands, G. Güntherodt, Phys. Rev. Lett. 74 (1995) 4476.

[10] H. Bethge, D. Heuer, Ch. Jensen, K. Reshöft, U. Köhler, Surf. Sci. 331 (1995) 878. 